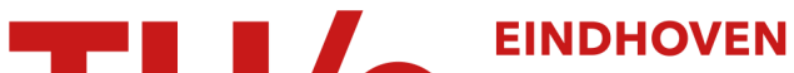 \\ UNIVERSITY OF \\ TECHNOLOGY
}

\section{Current-induced spin polarization at a single heterojunction}

\section{Citation for published version (APA):}

Silov, A. Y., Blajnov, P., Wolter, J. H., Hey, R., Ploog, K. H., \& Averkiev, N. S. (2004). Current-induced spin polarization at a single heterojunction. Applied Physics Letters, 85(24), 5929-5931.

https://doi.org/10.1063/1.1833565

DOI:

10.1063/1.1833565

Document status and date:

Published: 01/01/2004

\section{Document Version:}

Publisher's PDF, also known as Version of Record (includes final page, issue and volume numbers)

\section{Please check the document version of this publication:}

- A submitted manuscript is the version of the article upon submission and before peer-review. There can be important differences between the submitted version and the official published version of record. People interested in the research are advised to contact the author for the final version of the publication, or visit the DOI to the publisher's website.

- The final author version and the galley proof are versions of the publication after peer review.

- The final published version features the final layout of the paper including the volume, issue and page numbers.

Link to publication

\section{General rights}

Copyright and moral rights for the publications made accessible in the public portal are retained by the authors and/or other copyright owners and it is a condition of accessing publications that users recognise and abide by the legal requirements associated with these rights.

- Users may download and print one copy of any publication from the public portal for the purpose of private study or research.

- You may not further distribute the material or use it for any profit-making activity or commercial gain

- You may freely distribute the URL identifying the publication in the public portal.

If the publication is distributed under the terms of Article $25 \mathrm{fa}$ of the Dutch Copyright Act, indicated by the "Taverne" license above, please follow below link for the End User Agreement:

www.tue.nl/taverne

Take down policy

If you believe that this document breaches copyright please contact us at:

openaccess@tue.nl

providing details and we will investigate your claim. 


\title{
Current-induced spin polarization at a single heterojunction
}

\author{
A. Yu. Silov, ${ }^{\text {a) }}$ P. A. Blajnov, and J. H. Wolter \\ COBRA Inter-University Research Institute, Eindhoven University of Technology, PO Box 513, \\ NL-5600 MB Eindhoven, The Netherlands \\ R. Hey and K. H. Ploog \\ Paul-Drude-Institut für Festkörperelektronik, Hausvogteiplatz 5-7, 10117 Berlin, Germany \\ N. S. Averkiev \\ Ioffe Physico-Technical Institute, Polytekhnicheskaya 26, 194021 St. Petersburg, Russia
}

(Received 1 March 2004; accepted 18 October 2004)

\begin{abstract}
We have experimentally achieved spin polarization by a lateral current in a single nonmagnetic semiconductor heterojunction. The effect does not require an applied magnetic field. The current-induced spin orientation can be seen as the inverse of the circular photogalvanic effect (also often referred to as spin photocurrents): the nonequilibrium spin changes its sign as the current reverses. (C) 2004 American Institute of Physics. [DOI: 10.1063/1.1833565]
\end{abstract}

Today, there is a substantial interest in creating nonequilibrium spin polarization in semiconductors. While optical alignment of the spins developed into a very powerful technique already since the early 1970 s, $^{1}$ the electrical spin injection has became a hot topic of research only recently. Different schemes of the electrical injection were realized: through a paramagnetic spin aligner, ferromagnetic semiconductors, and ferromagnetic metal (for a comprehensive review of the recent results on electrical spin injection see Ref. 2). All these methods of electrical spin injection require an application of magnetic field or ferromagnetic contacts to achieve nonequilibrium spin polarization.

In this letter, yet another method of creating spin polarization is realized that differs radically from those employed so far. We have experimentally achieved the spin polarization that is induced by an electrical current alone, in the absence of applied magnetic field. Such spin polarization by an electric current can be seen as the inverse of the circular photogalvanic effect. In the photogalvanic effect, the angular momentum of the absorbed (circularly polarized) photon is transferred into a directed current of the charge carriers. The effect is allowed in quantum wells of III-V compounds due to the removal of the spin degeneracy. The extensive literature on spin photocurrents in low-dimensional structures is reviewed in Ref. 3.

In semiconductors with a strong spin-orbit interaction, an electric field acts on a moving charge carrier as an effective magnetic field and may be used to orient the spin. The current-induced spin orientation in semiconductors had been theoretically suggested by Dyakonov and Perel ${ }^{4}$ in the 1970s, but, up to now, was not realized experimentally. In lowdimensional systems (quantum wells and single heterojunctions), the theory of spin polarization in an electric field was developed in Refs. 5-7. In systems lacking inversion symmetry there exists an additional term of the spin-orbit interaction. That interaction is linear in the wave vector, $\boldsymbol{k}$, of charge carriers and manifests itself in lifting the spin degeneracy away from the zone center (see, for instance, Ref. 8). This can be caused either by (i) the inversion asymmetry of host material (Dresselhaus mechanism of spin splitting) or

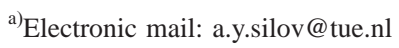

(ii) by the asymmetry of the confinement in low-dimensional systems (Bychkov-Rashba effect). Previously, the currentinduced spin polarization was theoretically considered only for the conduction electrons. However, the $\boldsymbol{k}$-linear terms are not unique for the conduction band only, and, in zinc blende semiconductors, are significantly larger in the valence band: the two-dimensional holes display much more pronounced removal of the spin degeneracy as compared to the electron gas in GaAs.

The sample under investigation comprises a single modulation-doped heterojunction to form the twodimensional hole gas (2DHG). The sample was synthesized by a molecular beam epitaxy on an exactly oriented $\mathrm{CrO}-$ doped semi-insulating (001) GaAs substrate. The layer sequence starts with a nominally undoped GaAs $(50 \mathrm{~nm})$ buffer layer followed by a 25 period GaAs/AlAs ( 5 by $5 \mathrm{~nm}$, respectively) superlattice. Subsequently, the active layer $(20 \mathrm{~nm})$ of undoped GaAs was deposited. On top of the active layer, a Be-doped $\left(1.33 \times 10^{18} \mathrm{~cm}^{-3}\right) \mathrm{Al}_{0.33} \mathrm{Ga}_{0.67}$ with a $20 \mathrm{~nm}$ spacer was grown. Finally, the $\mathrm{Al}_{0.33} \mathrm{Ga}_{0.67} \mathrm{As}$ layer was capped with a 8-nm-thick layer of GaAs. Annealed $\mathrm{Ni} / \mathrm{ZnAu}$ contacts were made to the $2 \mathrm{DHG}$ confined by the potential notch at the $\mathrm{Al}_{0.33} \mathrm{Ga}_{0.67} \mathrm{As} / \mathrm{GaAs}$ heterojunction. The hole concentration $\left(N_{\text {Hall }}\right)$ was $4.15 \times 10^{11} \mathrm{~cm}^{-2}$ with the mobility $\left(\mu_{\text {Hall }}\right)$ of $46500 \mathrm{~cm}^{2} / \mathrm{V} \mathrm{s}$ at $5.8 \mathrm{~K}$.

The nonequilibrium mean spin density is generally oriented in an arbitrary way in respect to the electric current. The angle between the mean spin and the electric current is governed by the interplay between the Dresselhaus and Bychkov-Rashba effects. It can be shown, for instance, that in the (311)-grown quantum wells the mean spin density will have a component along the growth direction. For the quantum wells on (001)-surface, however, the spin density has only planar components. ${ }^{7}$ To detect unambiguously currentinduced spin orientation, our experiments were done on the (001)-surface with the electric current along [0 $\overline{1} 1]$-direction (see inset in Fig. 1). In this case, from symmetry considerations, the mean spin density has only a planar component perpendicular to the current flow. And the interplay between Dresselhaus and Bychkov-Rashba terms will dictate an absolute value of the spin polarization, but not the spatial distribution. 


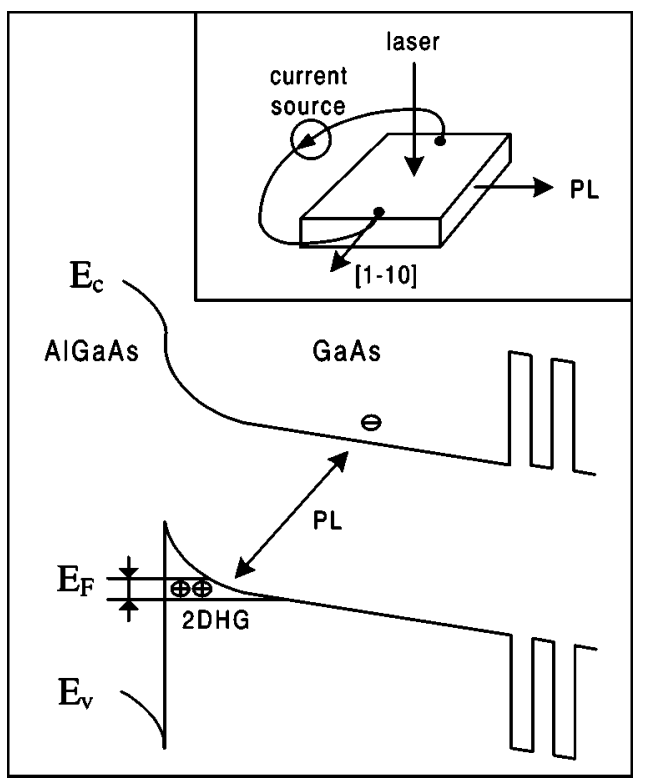

FIG. 1. Energy diagram sketch of a $p$-AlGaAs/GaAs single heterojunction. The arrow indicates the recombination of photoexcited electrons with the two-dimensional holes. The inset displays the experimental arrangement.

To detect the spin polarization, we have measured the degree of circular polarization, $P$, of the 2DHG photoluminescence, a method originally proposed for detecting the electrical spin injection through a ferromagnetic contact. ${ }^{9}$ This experimental procedure has become a proven method for probing spin polarization. ${ }^{2}$ The sample was cleaved into bars of $1 \times 2.5 \mathrm{~mm}^{2}$ with the current flowing along the long side, which is in the $[1 \overline{1} 0]$ direction. The samples were then mounted in a He-flow cryostat. The photoluminescence (PL) was excited with $633 \mathrm{~nm}$ line from a helium-neon laser. The laser was power stabilized with less than $0.2 \%$ output power fluctuation over $5 \mathrm{~h}$.

The PL was collected from the cleaved (110) facet of the sample. Detection of glancing light along the surface was prevented by detecting the PL through a narrow aperture on the cleaved facet. The degree of circular polarization $P$ $=\left(\sigma^{+}-\sigma^{-}\right) /\left(\sigma^{+}+\sigma^{-}\right)$was analyzed with a $\lambda / 4$ wave plate and linear polarizer. The base line of the circularly polarized PL was carefully checked by recording a difference between $\mathrm{PL}_{\sigma-}$ and $\mathrm{PL}_{\sigma+}$ spectra when no current was delivered through the sample. The inset in Fig. 1 shows the experimental arrangement for measuring the current-induced polarization. The current flow was stabilized with an accuracy of $50 \mathrm{pA}$. The PL was detected with an optical multichannel analyzer employing a liquid-nitrogen cooled charge-coupled device attached to a $0.35 \mathrm{~m}$ spectrometer.

Figure 2(a) shows the low-temperature PL spectra both with a current flow turned on or switched off. The PL contains a strong radiative band centred at about $1.52 \mathrm{eV}$. This band originates from recombination of the two-dimensional holes with electrons photoexcited into the GaAs conduction band. ${ }^{10}$ Figure 1 schematically depicts the 2DHG recombination process. Due to the many-body shake-up of the $2 \mathrm{DHG}$ Fermi sea, the radiative band has a clear asymmetrical spectral shape. The high-energy side of the spectra displays an abrupt intensity drop at the Fermi energy of 2DHG. The PL intensity is decreased by passing the current through the sample, while the emission wavelength is blueshifted. This spectral shift towards the higher energy side is explained by Downloaded 12 Nov 2007 to 131.155.108.71. Redistribution subject
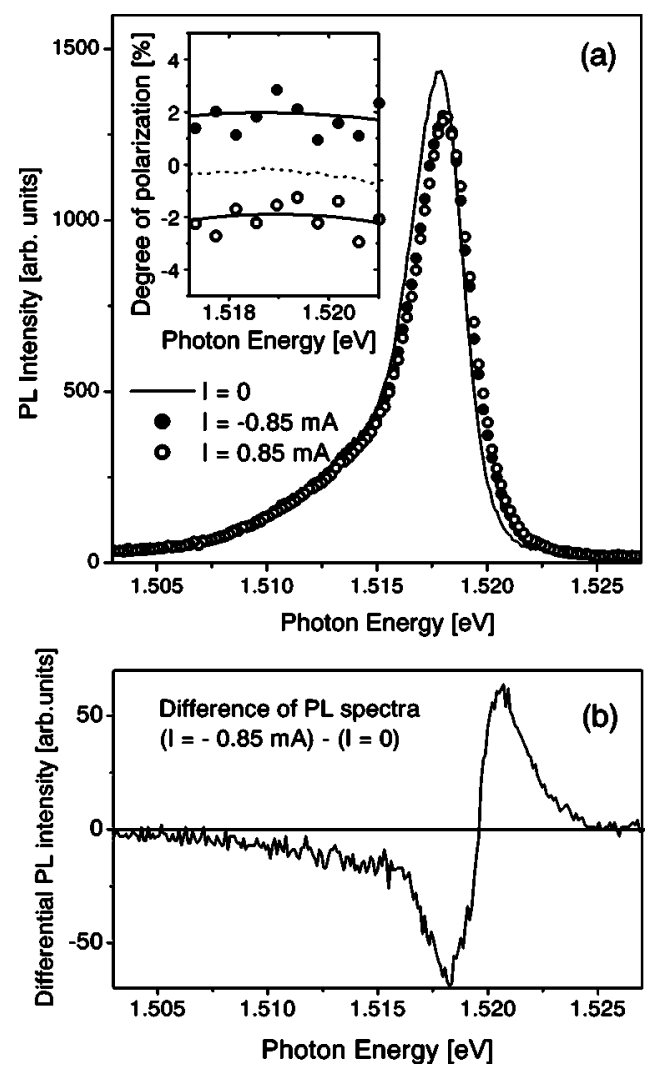

FIG. 2. Panel (a): Photoluminescence spectra at $5.1 \mathrm{~K}$ in absence of a current (solid line) and with current of $\pm 0.85 \mathrm{~mA}$ in opposite directions (empty and filled circles, respectively). Spectral resolution is $1.8 \mathrm{meV}$, excitation density is $1.2 \mathrm{~mW} / \mathrm{cm}^{2}$. Inset shows a degree of circular polarization within the full width at half maximum. The dotted line gives the polarization base line. The solid lines are a guide for the eye. Panel (b): Differential PL spectrum current on/off.

the extra energy acquired by the 2 DHG in the lateral electric field. This is different from Joule overheating by the current through the 2D channel: heating leads to a redshift of the PL band. ${ }^{11}$ As was pointed out in Ref. 6, current-induced spin polarization in the quantum wells may only occur as a result of spin relaxation of thermalized carriers. In the present experiment, the Joule heating was avoided by delivering less than $1.6 \mathrm{~mW}$ of dc power. Under these conditions, we always observe an effective blueshift, which is illustrated in Fig. 2(b). Figure 2(b) shows a differential PL spectrum when the PL signal without electric current was used as a background spectrum for the measurements when current was switched on. The differential spectra were measured in background substraction mode of the charge-coupled detector.

The inset in Fig. 2(a) shows the observed $P$ with the dotted line representing a base line of the circular polarization. The observed degree of polarization yields a maximum of $2.5 \%$. It is the important feature of the data that the degree of polarization inverses its sign as the electric current reverses. This is a direct prove of the current-induced spin orientation, in complete agreement with the results on the spin photocurrents in quantum wells. ${ }^{12}$ The change of sign in the degree of circular polarization is also illustrated by Fig. 3 where the differential spectra, $\left(\mathrm{PL}_{\sigma_{+}}-\mathrm{PL}_{\sigma-}\right)$, are plotted for the two opposing currents.

The spin orientation, $S$, can be estimated following calculations for 2D electrons from Ref. 6:

to AlP license or copyright, see http://api.aip.org/apl/copyright.jsp 


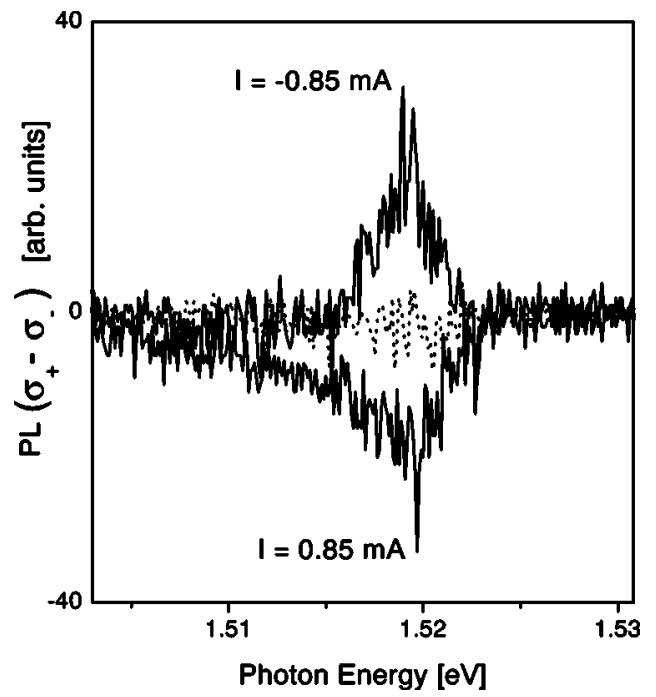

FIG. 3. Differential spectra of polarized PL. Base line (dotted curve) is taken with the current turned off.

$$
S=6 Q \frac{\gamma F e \tau_{p}}{\hbar E_{F}},
$$

where $Q$ is a coefficient of approximately unity depending of the scattering mechanism, $E_{F}$ is the DHG Fermi energy, $F$ is an electric field, $e$ is elementary charge, $\tau_{p}$ is the momentum relaxation time, and $\gamma$ describes the $\boldsymbol{k}$-linear spin splitting. In a field of $6.5 \mathrm{~V} / \mathrm{cm}$, with $\gamma=7 \times 10^{-2} \mathrm{eV} \AA$ (from linear interpolation of data), ${ }^{13} \tau_{p}=10^{-11} \mathrm{~s}$ and $E_{F}=2 \mathrm{meV}$ from our transport measurements, the spin polarization is estimated to be $10 \%$. Although in some cases the degree of circular polarization can be quantitatively linked to the spin polarization, ${ }^{14}$ for the $2 \mathrm{DHG}$ recombination in the [110] direction the link is not straightforward. It is only in the undoped quantum wells on (001) with sufficient splitting between heavy- and light-hole states that the degree of luminescence polarization along the growth direction is simply equal to the spin orientation. What is, however, essential for the cleaved-side arrangements is that the circularly polarized optical transition is not forbidden. ${ }^{12,15}$ It is the admixture of the light-hole character to the ground (heavy-hole) state of the 2DHG that makes detection of the planar mean spin density possible in our experiments. The experimentally observed $P$ of $2.5 \%$ yields only a low-limit estimate of the nonequilibrium spin-polarization. Nonetheless, even $S$
$=2.5 \%$ exceeds the spin polarization that has been initially achieved by the spin injection from ferromagnetic metals into GaAs. ${ }^{16}$

Although Eq. (1) suggests linearity of the effect on the electrical field, one should keep in mind that the 2 DHG represents a self-consistent system, with the confinement being changed as the current passes. This effect is beyond the scope of the present letter and will be discussed in detail elsewhere. The essential result of our experiments is that the degree of polarization represents an odd function of the electric current.

The authors thank R. W. van der Heijden and O. V. Kurnosikov for stimulating discussions, T. Campbell Rickets for his help in the initial stage of the experiments, and P. Nouwens for the fabrication of the samples. This work was financially supported by the Dutch Organization for Advancement of Research (NWO). The research at the Ioffe Institute was also supported by INTAS, RFBR, scientific programs of RAS and Russian Ministry of Industry, Science and Technology.

${ }^{1}$ Optical Orientation, edited by F. Meier and B. Zakharchenya (NorthHolland Physics, Amsterdam, 1984).

${ }^{2}$ Semiconductor Spintronics and Quantum Computation, edited by D. Awschalom, D. Loss, and N. Samarth (Springer, Berlin, 2002).

${ }^{3}$ S. D. Ganichev and W. Prettl, J. Phys.: Condens. Matter 15, R935 (2003).

${ }^{4}$ M. I. Dyakonov and V. I. Perel, Phys. Lett. 35A, 459 (1971).

${ }^{5}$ V. M. Edelstein, Solid State Commun. 73, 233 (1990).

${ }^{6}$ A. G. Aronov, Yu. B. Lyanda-Geller, and G. E. Pikus, Sov. Phys. JETP 73, 537 (1991).

${ }^{7}$ A. V. Chaplik, M. V. Entin, and L. I. Magarill, Physica E (Amsterdam) 13, 744 (2002).

${ }^{8}$ E. I. Rashba and V. I. Sheka, in Landau Level Spectroscopy, edited by G. Landwehr and E. I. Rashba (North-Holland, Amsterdam, 1991), Vol. 1, p. 178.

${ }^{9}$ G. E. Pikus and A. G. Aronov, Sov. Phys. Semicond. 10, 698 (1976).

${ }^{10}$ A. Yu. Silov, J. E. M. Haverkort, N. S. Averkiev, P. M. Koenraad, and J. H. Wolter, Phys. Rev. B 50, 4509 (1994).

${ }^{11}$ A. Yu. Silov, V. M. Asnin, N. S. Averkiev, J. E. M. Haverkort, L. M. Weegels, G. Weimann, and J. H. Wolter, J. Appl. Phys. 73, 7775 (1993).

${ }^{12}$ S. D. Ganichev, E. L. Ivchenko, S. N. Danilov, J. Eroms, W. Wegscheider, D. Weiss, and W. Prettl, Phys. Rev. Lett. 86, 4358 (2001).

${ }^{13}$ H. L. Störmer, Z. Schlesinger, A. Chang, D. C. Tsui, A. A. Gossard, and W. Wiegmann, Phys. Rev. Lett. 51, 126 (1983).

${ }^{14}$ B. T. Jonker, A. T. Hanbicki, Y. D. Park, G. Itskos, M. Furis, G. Kioseoglou, A. Petrou, and X. Wei, Appl. Phys. Lett. 79, 3098 (2001).

${ }^{15}$ D. K. Young, E. Johnston-Halperin, and D. D. Awschalom, Appl. Phys. Lett. 80, 1598 (2002).

${ }^{16}$ B. T. Jonker, Proc. IEEE 91, 727 (2003). 